Article

\title{
The Pan-and-Tilt Hyperspectral Radiometer System (PANTHYR) for Autonomous Satellite Validation Measurements-Prototype Design and Testing
}

\author{
Dieter Vansteenwegen $^{1, *}$, Kevin Ruddick ${ }^{2}$, André Cattrijsse ${ }^{1}$, Quinten Vanhellemont ${ }^{2}$ \\ and Matthew Beck ${ }^{2}$ iD \\ 1 Flanders Marine Institute (VLIZ), Wandelaarkaai 7, 8400 Ostend, Belgium; dre@vliz.be \\ 2 Royal Belgian Institute of Natural Sciences (RBINS), Operational Directorate Natural Environment, \\ 29 Rue Vautierstraat, 1000 Brussels, Belgium; kruddick@naturalsciences.be (K.R.); \\ qvanhellemont@naturalsciences.be (Q.V.); mbeck@naturalsciences.be (M.B.) \\ * Correspondence: dieter.vansteenwegen@vliz.be
}

Received: 17 May 2019; Accepted: 3 June 2019; Published: 6 June 2019

\begin{abstract}
This paper describes a system, named "pan-and-tilt hyperspectral radiometer system" (PANTHYR) that is designed for autonomous measurement of hyperspectral water reflectance. The system is suitable for deployment in diverse locations (including offshore platforms) for the validation of water reflectance derived from any satellite mission with visible and/or near-infrared spectral bands (400-900 nm). Key user requirements include reliable autonomous operation at remote sites without grid power or cabled internet and only limited maintenance (1-2 times per year), flexible zenith and azimuth pointing, modularity to adapt to future evolution of components and different sites (power, data transmission, and mounting possibilities), and moderate hardware acquisition cost. PANTHYR consists of two commercial off-the-shelf (COTS) hyperspectral radiometers, mounted on a COTS pan-and-tilt pointing system, controlled by a single-board-computer and associated custom-designed electronics which provide power, pointing instructions, and data archiving and transmission. The variable zenith pointing improves protection of sensors which are parked downward when not measuring, and it allows for use of a single radiance sensor for both sky and water viewing. The latter gives cost reduction for radiometer purchase, as well as reduction of uncertainties associated with radiometer spectral and radiometric differences for comparable two-radiance-sensor systems. The system is designed so that hardware and software upgrades or changes are easy to implement. In this paper, the system design requirements and choices are described, including details of the electronics, hardware, and software. A prototype test on the Acqua Alta Oceanographic Tower (near Venice, Italy) is described, including comparison of the PANTHYR system data with two other established systems: the multispectral autonomous AERONET-OC data and a manually deployed three-sensor hyperspectral system. The test established that high-quality hyperspectral data for water reflectance can be acquired autonomously with this system. Lessons learned from the prototype testing are described, and the future perspectives for the hardware and software development are outlined.
\end{abstract}

Keywords: Hyperspectral reflectance; validation; autonomous measurements; ground-truth data; system design

\section{Introduction}

The objective of this paper is to describe the motivation, design, and prototype testing for an autonomous system of hyperspectral radiometers suitable for validation of satellite-derived water reflectance. 


\subsection{Motivation and Objective}

Satellite imagery of marine, coastal, and inland water reflectance is now routinely used for measuring parameters such as the concentrations of chlorophyll a, a proxy for phytoplankton biomass, and suspended particulate matter, important for sediment transport applications. The satellite data are used for regulatory monitoring of the aquatic environment, e.g., via the European Union Water Framework and Marine Strategy Framework Directives [1,2], and for providing a scientific basis for coastal zone decision-making, e.g., via the assessment of impacts of human activities and constructions [3]. However, end-users of the data require reliable information on data quality, and validation of the satellite data at the level of water reflectance is particularly crucial. This is because of the large errors that may occur during the data calibration and processing, particularly during the atmospheric correction steps [4].

This validation is best achieved by a "match-up" of in situ measurements of water (surface) reflectance made at the same time as the satellite measurement [5], and experience over the last 10 years showed that only autonomous in situ systems can provide sufficient data for this purpose. In particular, AERONET-OC [6], a federated network of multispectral robotically pointed radiometers on offshore platforms all over the world, proved to be the main source of validation data [7] for spaceborne optical missions such as ENVISAT/MERIS, MODIS/AQUA, VIIRS, Sentinel-3/OLCI, Sentinel-2/MSI, Landsat-8/OLI, etc. However, the radiometer adopted within AERONET-OC is only multispectral and cannot adequately cover the spectral bands of all recent and future optical spaceborne missions without spectral interpolation/extrapolation/modelling [8] and associated uncertainties. The WATERHYPERNET network is, therefore, being developed, based closely on the concept of the successful AERONET-OC network [6] but with the essential advantage of a hyperspectral radiometer, thus enabling the validation of all visible and near-infrared bands of all present and future satellite missions providing water reflectance data.

The objective of the present paper is to describe the measurement system, called PANTHYR (pan-and-tilt hyperspectral radiometer) that was developed for use within the WATERHYPERNET network. This measurement system consists of two commercial off-the-shelf (COTS) hyperspectral radiometers, mounted on a COTS pan-and-tilt (PT) pointing system, controlled by a single-board-computer and associated custom-designed electronics which provide power, pointing instructions, and data archiving and transmission.

\subsection{Measurement System Requirements}

The PANTHYR system was developed to fit the following user requirements:

- Measurement of downwelling irradiance, as well as downward (sky) and upward (water) radiance just above the water surface, at flexible zenith and azimuth (relative to sun) angles for a spectral range covering at least 400-900 $\mathrm{nm}$ with full-width half-maximum (FWHM) spectral resolution of $10 \mathrm{~nm}$ or better and spectral sampling every $5 \mathrm{~nm}$ or better.

- Storage of all measurements and diagnostic logs and regular transmission to a land-based server.

- User interface with flexibility for scientists to easily program pointing and data acquisition scenarios.

- Reliable autonomous operation at remote sites, e.g., offshore platforms, with a typical maintenance frequency of once or twice per year without grid power.

- Resistance to harsh offshore environments, including large temperature ranges (measurement limited to between $2{ }^{\circ} \mathrm{V}$ and $40^{\circ} \mathrm{C}$, and survival between $-20^{\circ} \mathrm{C}$ and $60^{\circ} \mathrm{C}$ ambient temperature), rain, salty sea spray, atmospheric deposition, and possible animals (birds, spiders, etc.).

- Modularity to adapt to sites with different possibilities for power (grid/solar/wind), data transmission channels (cabled internet, 3G/4G cellular networks), and mechanical mounting possibilities (rails, masts, etc.), and to cope with future evolution of system components.

- Moderate hardware purchase costs, e.g., typically $<10,000 €$ commercial price excluding taxes for a full system excluding radiometers. 
- Pointing accuracy of at least $5^{\circ}$ azimuth and $1^{\circ}$ zenith.

\subsection{Precursor Autonomous Systems}

A few autonomous systems with pointable radiometers already exist and are briefly described here.

The most used autonomous system for measurement of water reflectance is the Seaprism version of the CIMEL CE318 multiband photometer system. This system was originally commercialized in the 1990s for the measurement of aerosol properties and includes direct sun, near sun, and principal plane and almucantar sky radiance measurements, and it became the unique instrument of the AERONET network [9]. The Seaprism version was developed in the early 2000s [10] by reprogramming of the pointing system, especially to perform downward pointing measurements of upwelling radiance from water. The system evolved over the years to include improvements of the optical and electronic components, as well as development of new versions, e.g., including the possibility of nighttime lunar measurements [11]. The system consists essentially of an optical head with two fore-optics protected by collimators, containing a filter wheel for multiband optical measurements with a very wide dynamic range (from direct sun to dark water), a robotic pointing system, a control box providing pointing and measurement instructions and managing power and data, and associated auxiliary equipment for power generation (solar panels) and data transmission (METEOSAT satellite network uplink or cellular link). The system is extremely robust, giving reliable maintenance-free performance for long-term ( 1 year) deployments in a very wide range of environments including land and water sites from the tropics to the polar regions.

The OSPREY system [12] was designed to be commercialized by Biospherical Instruments as a very-high-performance modular system of radiometers on pointing systems. The radiometers include a hyperspectral spectrometer and numerous single-band microradiometers within a single thermally controlled casing and a filter wheel in front of the spectrometer allowing polarimetric, direct sun, straylight-corrected, and dark measurements. The system, described in detail in Reference [12], has a very high dynamic range and high accuracy pointing, and is capable of water, sun, sky, and moon radiance measurements.

The RFLEX system [13] consists of three hyperspectral TRIOS/RAMSES radiometers measuring downwelling irradiance, downwelling sky radiance, and upwelling water radiance mounted at fixed zenith angles $\left(0^{\circ}, 40^{\circ}\right.$, and $140^{\circ}$, respectively) on an azimuthally rotating platform with associated control software. This system was designed principally for deployment on moving ships, where the azimuthally rotating platform allows achieving an optimal $90^{\circ}$ or $135^{\circ}$ relative azimuth to sun for any ship heading. Instructions for system hardware construction using low-cost components (except for the COTS radiometers) were made publicly available via SourceForge (https://sourceforge.net/projects/rflex/) and source code for communication with TRIOS/RAMSES was made available via GitHub (https: //github.com/StefanSimis/PyTRIOS). The system was used operationally on two "ferryboxes" mounted on ships of opportunity in the Baltic Sea.

The DALEC system [14] is commercially available from Insitu Marine Optics and consists of three hyperspectral spectrometers measuring downwelling irradiance, and downwelling (sky) and upwelling (water) radiance embedded at fixed zenith angles $\left(180^{\circ}, 140^{\circ}\right.$, and $40^{\circ}$, respectively) within a compact azimuthally rotating body on a gimballed mount. Control and data are managed by a standard personal computer (PC) using software supplied by the manufacturer. This system was designed principally for deployment on moving ships but can also be used from fixed platforms. The system is deployed on research vessels and an example of data usage is described by Reference [15].

The SAS Solar Tracker system from Seabird Electronics consists of three hyperspectral Seabird/HyperSAS radiometers measuring downwelling irradiance, downwelling sky radiance, and upwelling water radiance mounted at fixed zenith angles $\left(180^{\circ}, 140^{\circ}\right.$, and $40^{\circ}$, respectively) on an azimuthally rotating platform, with compass-based sun-tracking to ensure optimal relative azimuth angle with respect to sun. Power and data are managed via a deck interface unit and a standard PC. The system is used routinely on a few research vessels and ships of opportunity [16]. 
The WISP-3 is a handheld system, commercially available from Water Insight, with three embedded radiometers measuring water reflectance from downwelling irradiance, sky radiance, and water radiance. Data for monitoring parameters such as chlorophyll a concentration are logged internally and can be downloaded to a PC as processed data. A variant called WISPstation is under development for autonomous operation. An example of data usage and inter-comparison with other systems is described in Reference [17].

The current PANTHYR design will provide hyperspectral reflectance data not available from the Seaprism system and has advantages over the RFLEX, DALEC, Suntracker, and WISP designs because the zenith pointing flexibility allows sky and water radiance measurements to be made by the same radiometer and allows downward pointing to protect instruments when not measuring. Use of a single radiance sensor provides cost saving and reduces the uncertainties associated with spectral and radiometric calibration differences of the two-radiance-sensor systems. The disadvantage of the PANTHYR sequential measurement of water and sky radiance as compared to the simultaneous measurements possible with the two-radiance-sensor systems is considered to be minimal for the good clear sun and sky illumination conditions needed for satellite validation. If illumination conditions are suboptimal, this will be detected by the PANTHYR replicate measurements and will lead to data rejection. The PANTHYR system does not pretend to achieve the very high performance expected of the OSPREY system, but represents a much lower-cost alternative which should be ideal for deployment at multiple sites worldwide by organizations with moderate budgets.

All of these systems, including PANTHYR, are developing within a context where hyperspectral spectrometers are rapidly evolving with reduction of size, power, and cost (thanks to mass production for medical and industrial applications), and where pointing systems are becoming more affordable and more easily available as COTS items (thanks to mass production for applications such as video surveillance). Other technologies facilitating PANTHYR development include the massive improvements and cost reductions in data transmission (thanks to mobile phone networks) and in microcomputers.

\subsection{Overview of Paper}

In this paper, the design choices are described in Section 2, together with details of the control electronics, where most of the original developments were made, and the data acquisition protocol and data processing steps, which are strongly based on precursor systems. A first seaborne test of the system on Acqua Alta Oceanographic Tower (AAOT) is described in Section 3, where data acquisition and processing are also described for two precursor systems used for comparison, a manually supervised three-sensor hyperspectral system and the autonomous multispectral AERONET-OC system. Results from the prototype tests are described in Section 4. General system performance is evaluated with description of lessons learned for system improvements. A comparison is made between data acquired with PANTHYR and data from the two precursor systems. Finally, conclusions from these prototype tests and future perspectives for system refinement are outlined in Section 5.

\section{System Design}

\subsection{Top-Level Design Choices}

To stay within the design and future purchasing budget, we decided to use mostly COTS components. Self-design of all hardware would allow for greater optimization, but design and manufacturing of all components would add cost and complexity beyond the scope of our goals. Only when commercial hardware was not available or sufficient did we create our own. As availability of the chosen components will change in the next few years, special care was taken to implement a modular design in hardware and software. Individual component changes will require updated software/hardware for only that part, while most of the system remains the same. This also facilitates adding extra hardware and capabilities in the future. 
The TRIOS/RAMSES COTS hyperspectral radiometer was chosen because of its relatively high performance, highly robust and mature low-power design, and moderate price. This radiometer provides measurements for a spectral range of $320-950 \mathrm{~nm}$ and was used for satellite validation since 2002 [18] by many teams worldwide. The performance of this class of radiometer was extensively studied and characterized with regard to straylight [19], thermal sensitivity [20], polarization sensitivity [21], angular response of the irradiance units [22], and non-linearity. A low-cost, portable, LED-based FieldCAL device is available for rapid checking of radiometric sensitivity in the field.

The FLIR PTU-D48E COTS pointing system was chosen for the PANTHYR system because of its high performance (including good pan and tilt speeds and pointing accuracy), robust design, and moderate price.

While COTS rugged PCs are widely available, a key early decision was to use a small low-power embedded computer board with self-design of additional electronics for managing power and connectivity to components such as the radiometers, GNSS (Global Navigation Satellite System) receiver, and auxiliary sensors. This required more development time, but provided more control over power management and greater flexibility in connecting and supporting additional external devices.

The Linux operating system was chosen to facilitate software portability to next-generation hardware platforms and to improve reliability and security. Linux is open source, easy to customize, and ideal for an offshore system where logistics make on-site intervention difficult and/or expensive.

Python was chosen as the main programming language as it is available under an open-source license, is well known in the scientific community, and produces clear, readable code.

\subsection{System Overview and Key Components}

The overall PANTHYR system is outlined in Figure 1, showing the main hardware elements and the associated power and data connections. A small embedded computer board controls all components and forms the heart of the system. After start-up, a GNSS receiver provides UTC (Coordinated Universal Time), as well as location, allowing the system to calculate the position of the sun. An optional IP network camera can take still pictures from the measurement target areas, allowing users to check any suspect data for unusual conditions such as floating vegetation or debris, boats, birds, and other obstructions in the field of view. During a measurement cycle, the controller calculates the head position for each measurement step, points the instrument in the required zenith and azimuth angles, and makes a measurement. At the end of a measurement cycle, the head moves to a "park" position where the instruments are pointed downward to prevent fouling. The system then goes into a sleep state, conserving power while waiting for the next cycle.

A myriad of single-board computers with ever increasing performance became available on the market in the last few years. These small (around credit-card size) and cheap $(<100 €)$ boards manage to run an operating system and have on-board storage, as well as a network connection, while consuming as little as $0.5 \mathrm{~W}$ of power in standby. For our application, the BeagleBone Black has the following advantages:

- It has five serial ports, which allows it to interface directly with the instruments, without the need for additional external interfaces.

- It was proven to be a reliable option in industrial applications [23] and scientific research [24].

- It is readily available from electronic parts distributors.

To connect and interface the controller to the rest of the system, we designed two electronic boards which are plugged on top of the BeagleBone. The first board translates the voltage levels of the serial ports to RS-232-compatible levels so that the controller can communicate with the instruments and GNSS receiver. The second board provides protection and filtering on the power lines, as well as allowing power saving by cutting the power to external devices when they are not in use. Five protected power outputs with a maximum power draw of $2 \mathrm{~A}$ each can be controlled from software. An external solid-state relay is used to control power to the head, which is the only component that needs a switched $24 \mathrm{~V}$ direct current supply. 


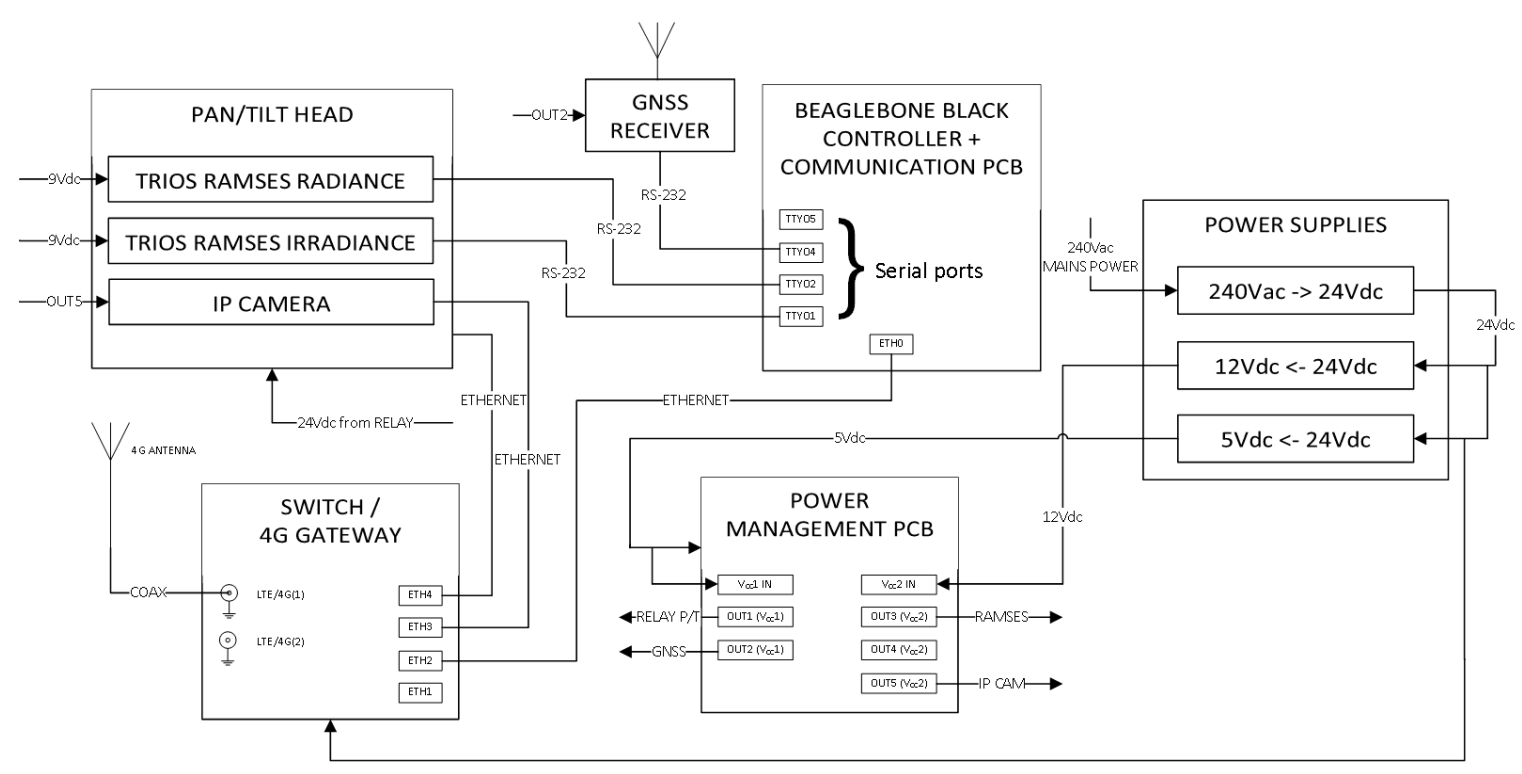

Figure 1. Pan-and-tilt hyperspectral radiometer system (PANTHYR) system diagram.

A number of commercially available PT heads were evaluated. We looked at price, resolution, power consumption, and ruggedness. The FLIR PTU-D48 E matched our requirements most closely. This unit was developed as a rugged solution for demanding applications and has the necessary specifications and certifications such as temperature range, ingress protection 67 , and salt spray protection (MIL-810G). Its maximum payload of $7.5 \mathrm{~kg}$ leaves margin for extra hardware.

To allow the full pan $\left(+/-174^{\circ}\right)$ and tilt $\left(+90^{\circ} /-30^{\circ}\right)$ movement, a free loop of cable is required between the rotating instruments and the base of the head. We found no good solution to prevent this loop of cables from getting in front of the instruments at certain positions. The next version of PANTHYR will use the same head but with built-in slip rings, providing internal connections in the head assembly to replace outside cables. This option also adds $360^{\circ}$ azimuth movement capability to the head. The limited tilt range of the PTU-D48 E means that the irradiance sensor which needs to be vertical during measurements cannot be parked lower than $30^{\circ}$ below the horizon; it is thought that this will provide adequate protection from the elements, but longer-term testing is required to confirm this. The limited tilt range $\left(120^{\circ}\right)$ of the PTU-D48 E also precludes an arrangement with parallel radiance and irradiance sensors since the radiance sensor would need to be tilted through $140^{\circ}$ to go from vertical (zenith $180^{\circ}$ ) to the necessary water-viewing angle (zenith $40^{\circ}$ ). The radiance sensor was, therefore, fixed at an angle of $40^{\circ}$ to the irradiance sensor, giving a zenith angle range for the irradiance sensor of $180^{\circ}$ (downwelling irradiance measurement) to $60^{\circ}$ (parked) and a zenith angle range for the radiance sensor of $140^{\circ}$ (sky radiance measurement) through $40^{\circ}$ (water radiance measurement) to $20^{\circ}$ (parked).

On average, the PT unit consumes $12.3 \mathrm{~W}$ during start-up, $6.2 \mathrm{~W}$ during hold, and up to $20 \mathrm{~W}$ during combined axial movements. This demonstrates the necessity of keeping the system in a low-power sleep mode as much as possible.

The InSYS MRO-L200 gateway/switch connects all Ethernet devices, as well as providing the gateway to a $4 \mathrm{G}$ cellular network. This industrial device can set up and manage a VPN connection to the onshore server, and has a serial port and digital output, which could be used to remotely power cycle PANTHYR in case of problems. Its high power consumption ( $2.5 \mathrm{~W}$ typical) is the main drawback. A low-power network switch and a separate $4 \mathrm{G}$ gateway that is switched on only during short intervals would mitigate this, but also remove our option to connect to the installation in case of problems.

Two mechanical structures were designed to mount to existing structures at the measurement sites and install the instruments. A versatile mounting system accommodates different measurement sites, while remaining simple. We started with a $50-\mathrm{mm}$ steel pipe as a range of clamps are commercially available for this standard diameter. Welded on top of this pipe is a triangular plate. The head is 
mounted on a second similar plate. Three threaded rods connect both plates atop each other. Leveling of the head is achieved by changing the spacing along the three rods. The result is a simple system with a variety of mounting options (Figure 2a).

On top of the head, both sides of a U-shaped bracket accept adapter plates that hold the radiometers. Bolting holes for fixed inclinations of $20^{\circ}$ and $60^{\circ}$, as well as a slot that allows a variable $10^{\circ}$ to $70^{\circ}$ inclination, are available (Figure 2b).

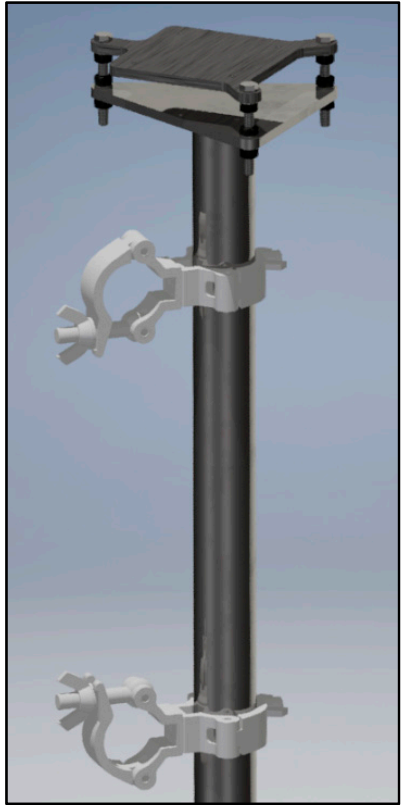

(a)

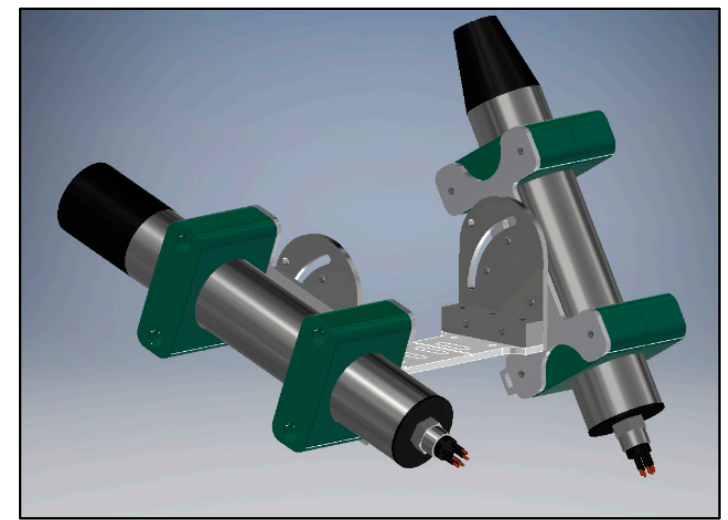

(b)

Figure 2. Engineering drawings of mounting structures. (a) Support providing a flexible connection between a platform structure, e.g., horizontal railings or a vertical post fixed through the swivel couplings, and pan/tilt unit to be mounted on uppermost horizontal surface; (b) sensor mounting bracket for attaching radiometers to top of pan/tilt unit.

\subsection{Software and Usage}

The main Python script checks system and user settings and controls all actions. Data and settings are stored in an SQLite database. Software will be released under the GNU GPLv3 (GNU's Not Unix General Public License version 3) at the GitHub page: www.github.com/hypermaq/panthyr.

PANTHYR has one SQLite database which contains the measurement protocol, system settings, logs, and measurement results, as well as a task queue. The user can use a (remotely) connected laptop to access a webpage where the contents of the database can be viewed, changed, and exported (Figure 3). This serves as the main user interface for system configuration.

A "worker script" regularly checks the "queue" database table for tasks that need to be performed. These tasks range from executing a measurement cycle to setting up station parameters.

To perform a measurement cycle, the system gets the necessary settings from the "settings" table and read the first line in the "protocol" table. Each line in this table contains the parameters (instrument, zenith angle, azimuth offset, number of repetitions) describing one sub-cycle in the measurement cycle. Measurement results are written to the "measurements" table. When all scans in the protocol are finished, the system resumes a standby state where it regularly checks for new tasks. In the event of a failure, a log entry is created in the "logs" table. After three failed attempts, a task is ignored to prevent it from blocking the system.

Apart from the database frontend, PANTHYR also hosts an FTP (File Transfer Protocol) server to download log files and pictures, as well as an SSH (Secure SHell) server to allow low-level system maintenance. To prevent provider restrictions enforced on cellular networks, PANTHYR initiates 
an outgoing OpenVPN connection to an onshore server. Users can connect to the same server and communicate with PANTHYR as if it were next to them.

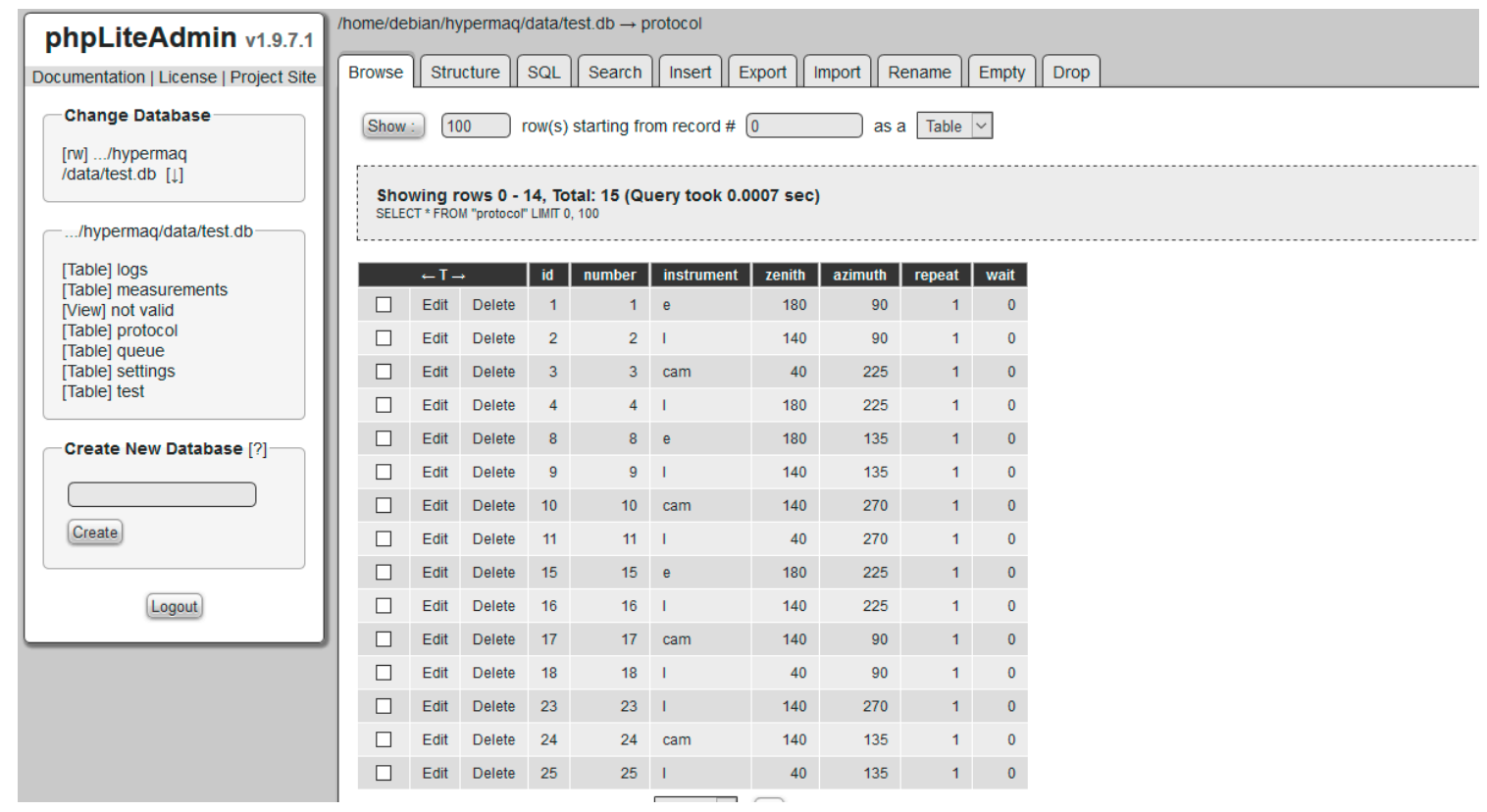

Figure 3. Screenshot of SQLite database interface.

\subsection{PANTHYR Data Acquisition Protocol}

The measurement protocol presented here is based on various precursors including References $[6,25,26]$ and, as usual for above-water radiometry, gives a water-leaving radiance or reflectance for an off-nadir geometry, which can be matched to satellite viewing geometries by appropriate models of a bidirectional reflectance distribution function. Research to determine an optimal measurement protocol for PANTHYR operations within the WATERHYPERNET network is in progress and some aspects may change in the future, e.g., potential additional zenith and/or azimuth angles and/or a different number of replicate measurements, especially for water viewing. However, the current protocol is considered as already sufficient for demonstrating performance of the hardware.

The PANTHYR system performs automated measurements every 20 min from sunrise until sunset. Each cycle consists of measurements with a $90^{\circ}, 135^{\circ}, 225^{\circ}$, and/or $270^{\circ}$ relative azimuth to sun. In general, and depending on the installation location, platform geometry, time of day (sun location), and associated platform shading of the water target, only one or two (or sometimes zero) of these azimuth angles are appropriate for measurement of water reflectance; other azimuth angles will be contaminated by platform shading or even direct obstruction of the water target as defined from the instrument field of view. A selection of acceptable azimuth angles is made a priori based on expert judgement (used here for prototype testing; see Section 3) or, better, a study of platform shading effects by modeling or experimentation [27]. The measurement of unacceptable azimuth angles, defined by a "no-go zone", can then be avoided to save power.

For each measurement cycle, the system performs a sub-cycle for each of the configured relative azimuth angles as described in Table 1. Based on the AERONET-OC protocol [6], but with repetition of the $E_{d}$ and $L_{d}$ replicates, each azimuthal measurement sub-cycle consists of $2 \times 3$ replicate scans each of downwelling irradiance, $E_{d}$, and sky radiance, $L_{d}$, and 11 replicate scans of upwelling radiance, $L_{u}$, where "(spectral) scan" refers to acquisition of a single instantaneous spectrum. Firstly, the irradiance sensor is pointed upward, with the radiance sensor offset by $40^{\circ}$, and three replicates of $E_{d}$ followed by three replicates of $L_{d}$ are measured. The radiance sensor is then moved to a $40^{\circ}$ downward viewing angle to make 11 replicate $L_{u}$ scans. The irradiance and radiance sensors are then repositioned to make 
three more replicate scans each of $L_{d}$ and $E_{d}$. Scans are stored as digital counts (DCs), including the embedded dark pixel counts, with instrument serial number and integration time in the metadata.

Table 1. Pan-and-tilt hyperspectral radiometer system (PANTHYR) basic data acquisition cycle. Azimuth is measured clockwise with respect to sun with a relative azimuth of $0^{\circ}$, meaning that the radiance sensor is pointing toward the sun, and a relative azimuth of $90^{\circ}$, meaning that the radiance sensor is pointing to the right of sun. A zenith angle of $180^{\circ}$ means that the instrument is pointing vertically upward and, hence, measuring downwelling (ir)radiance. Measurements within a predefined azimuth no-go zone will be skipped.

\begin{tabular}{cccccc}
\hline $\begin{array}{c}\text { Sub-Cycle } \\
\text { Number }\end{array}$ & Instrument & Measurement & $\begin{array}{c}\text { Zenith } \\
\text { Angle }\left(^{\circ}\right)\end{array}$ & $\begin{array}{c}\text { Azimuth Relative } \\
\text { to Sun }\left(^{\circ} \text { ) }\right.\end{array}$ & $\begin{array}{c}\text { Replicate } \\
\text { Scans }\end{array}$ \\
\hline 1 & Irradiance & $E_{d}$ & 180 & 90 & 3 \\
2 & Radiance & $L_{d}$ & 140 & 90 & 3 \\
3 & Camera & Sky photo & 140 & 90 & - \\
4 & Radiance & $L_{u}$ & 40 & 90 & 11 \\
5 & Camera & Water photo & 40 & 90 & - \\
6 & Radiance & $L_{d}$ & 140 & 90 & 3 \\
7 & Irradiance & $E_{d}$ & 180 & 90 & As 1-7 \\
$8-14$ & As 1-7 & As 1-7 & As 1-7 & 135 & As 1-7 \\
$15-21$ & As 1-7 & As 1-7 & As 1-7 & 225 & As 1-7 \\
$22-28$ & As 1-7 & As 1-7 & As 1-7 & 270 & \\
\hline
\end{tabular}

\subsection{PANTHYR Data Processing}

Each of the scans within a sub-cycle are converted from DCs to calibrated (ir)radiances, as described in Equations (1-3) of Reference [20], using the calibration files appropriate for the instrument serial number and date as follows:

- $\quad$ The 16-bit DCs are normalized by dividing by 65,535 .

- A long-term dark current correction is performed taking into account the instrument factory characterization and the scan integration time.

- A residual dark signal is subtracted using the mean average from the sensor dark pixels.

- The signal is normalized by the integration time and divided by the calibration sensitivity to retrieve final calibrated (ir)radiances.

Incomplete spectra are removed, as well as $L_{d}$ and $L_{u}$ scans with $>25 \%$ difference between neighboring scans at $550 \mathrm{~nm}$. $E_{d}$ scans are removed using the same criterion after normalizing $E_{d}$ by $\operatorname{Cos}\left(\theta_{0}\right)$, where $\theta_{0}$ is the sun zenith angle. The sub-cycle is further processed if sufficient scans pass the quality control: $L_{u} 9 / 11$, and $E_{d}$ and $L_{d} 5 / 6$ each. The remaining $E_{d}$ and $L_{d}$ measurements are then grouped and mean averaged to $\overline{E_{d}}$ and $\overline{L_{d}}$. For each $L_{u}$ scan, the water-leaving radiance, $L_{w}$, is computed by removing skyglint radiance as follows:

$$
L_{w}=L_{u}-\rho_{F} \overline{L_{d}}
$$

where $\rho_{F}$ is the "wind-roughened Fresnel" coefficient that represents the fraction of incident skylight that is reflected back toward the water-viewing sensor at the air-water interface and is given by the look-up table (LUT) of Reference [25]. This LUT describes $\rho_{F}$ as function of viewing and sun geometry and wind speed. Wind speed is retrieved from ancillary data files or set to a user-defined default value if wind speed data are unavailable. The data in the LUT are linearly interpolated to the current observation geometry and wind speed.

The $L_{w}$ scans are then converted into ("uncorrected") water-leaving radiance reflectance scans, $\rho_{w u}$, as follows:

$$
\rho_{w u}=\pi \frac{L_{w}}{\overline{E_{d}}} .
$$


A "near infrared (NIR) similarity spectrum" correction is then applied to remove any white error from inadequate skyglint correction as shown in Equation (3). A spectrally flat measurement error, $\varepsilon$, is estimated using two wavelengths in the NIR [28], where $\lambda_{1}=780 \mathrm{~nm}$ and $\lambda_{2}=870 \mathrm{~nm}$.

$$
\varepsilon=\frac{\alpha \rho_{w u}\left(\lambda_{2}\right)-\rho_{w u}\left(\lambda_{1}\right)}{\lambda_{2}-\lambda_{1}},
$$

where $\alpha$ is the similarity spectrum [26] ratio for the bands used; here, $\alpha(780,870)=1 / 0.523=1.912$. Per scan, $\varepsilon$ is subtracted from the $\rho_{w}$ at all wavelengths to give an NIR-corrected water reflectance,

$$
\rho_{w}(\lambda)=\rho_{w u}(\lambda)-\varepsilon,
$$

and all $\rho_{w}$ scans are mean-averaged to give the final NIR-corrected water reflectance, $\overline{\rho_{w}}$. This NIR correction is optional and may be turned off, e.g., for extremely turbid waters where it is not valid and/or for situations where the satellite measurement may include a similar constraint in the atmospheric correction which needs to be validated independently.

The final quality control to retain or reject the $\rho_{w}$ is performed according to Reference [26]; measurements are rejected when $\overline{L_{d}} / \overline{E_{d}}>0.05 \mathrm{sr}^{-1}$ at $750 \mathrm{~nm}$ (indicating clouds either in front of the sun or in the sky-viewing direction), and when the coefficient of variation $(\mathrm{CV}$, standard deviation divided by the mean) of the $\rho_{w}$ scans is $>10 \%$ at $780 \mathrm{~nm}$.

\section{Prototype Testing}

\subsection{July 2018 Deployment at the Acqua Alta Oceanographic Tower}

After extensive testing of individual components and the full system in the laboratory and outdoors on land, a first prototype system test was performed on the Acqua Alta Oceanographic Tower (AAOT) in July 2018 during the international Field Inter-Comparison Exercise (FICE) organized by the FRM4SOC (Fiducial Reference Measurements for Satellite Ocean Colour) project. AAOT is an oceanographic platform in the Northern Adriatic Sea $15 \mathrm{~km}$ offshore from Venice in water of $16 \mathrm{~m}$ depth. It was used extensively over the last 20 years for oceanographic data collection including optical oceanography [29] and was used for a number of multi-partner optical radiometry inter-comparison exercises [30,31]. It is the location of the first AERONET-OC site and provides such data since 2002. The above-water platform structure was entirely reconstructed in 2018, but the new structure closely follows the design and layout of the original platform.

The PANTHYR system was deployed on the east corner of the top deck of the platform (Figure 4), with the irradiance sensor collector $2 \mathrm{~m}$ above top deck floor and, hence, about $14 \mathrm{~m}$ above sea level. The system ran for six days continuously from 12 to 17 July 2018, under the supervision of the developer for the first three days and with non-specialized supervision for the remaining days. Some test conditions were not the most challenging that can be expected in the future; grid power was available, and data were not transmitted over a $4 \mathrm{G}$ link but stored onboard. However, the basic functions of autonomous pointing and data acquisition were fully and successfully tested.

This deployment for prototype testing was also installed only at moderate height and not above the height of the top deck masts for practical reasons; for future operational deployments, the PANTHYR system should ideally be positioned above the height of all such masts and structures or with a careful analysis of any shading of downwelling irradiance. For subsequent data comparison, only PANTHYR measurements at relative azimuth angles of $225^{\circ}$ or $270^{\circ}$ were used.

Using the angular field-of-view data ( $7^{\circ}$ full-width half-maximum) for the radiance instrument supplied by the manufacturer suggests that the water-viewing radiometer sees an elliptic patch of water with about $3 \mathrm{~m}$ diameter. Typical integration time for the water-viewing measurement is $\leq 512$ ms during this experiment. Spatio-temporal variability caused by the surface wave field is, thus, mainly averaged and not resolved. 
The FICE campaign further involved the deployment of multiple supervised radiometer systems, including seven above-water radiometer systems, one shipborne free-fall underwater radiometer system, and one platform-deployed vertical profiling system. Inter-comparison of all these systems will be the focus of a separate paper. In the current paper, results of the PANTHYR system are compared only to one supervised above-water radiometer system, the manually supervised RBINS (Royal Belgian Institute of Natural Sciences) hyperspectral above-water three-radiometer TRIOS/RAMSES system, hereafter called the "M3TRIOS" system, and the autonomous multispectral AERONET-OC system operated by the Joint Research Centre (JRC) of the European Commission at Ispra. The five TRIOS/RAMSES sensors used here (two for PANTHYR, three for M3TRIOS) were radiometrically calibrated at the Tartu Observatory laboratory just before the FICE campaign, thus minimizing calibration-related differences.

Only measurements from the five days with best sky conditions (13-17 July) are used here. For those days and during the period of day used for comparison in the present study, sun zenith angles ranged from $23.4^{\circ}$ to $46.7^{\circ}$ and wind speed ranged from 0.1 to $4.3 \mathrm{~m} / \mathrm{s}$.

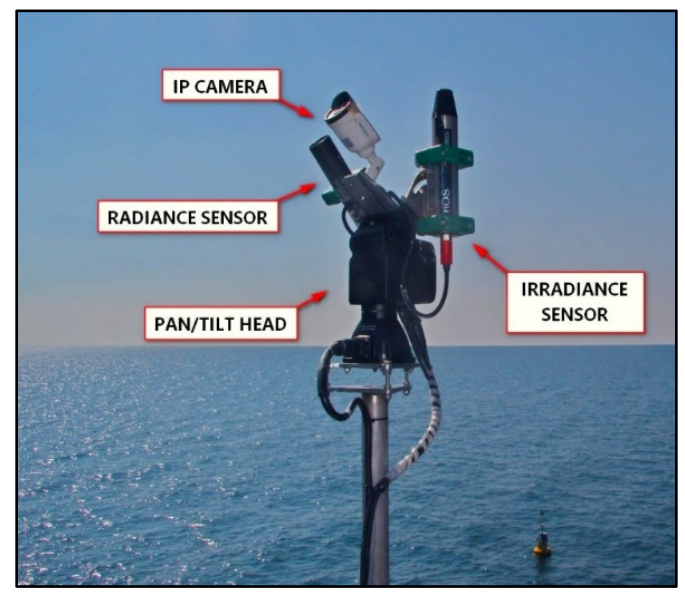

(a)

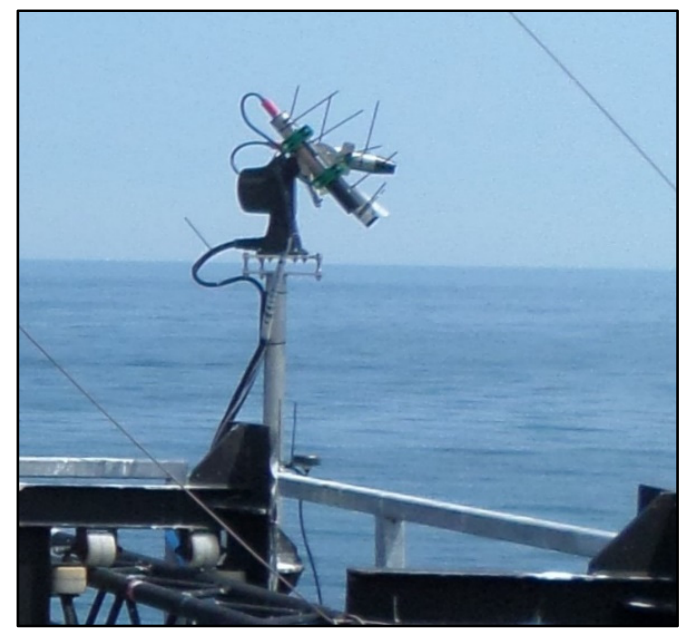

(c)

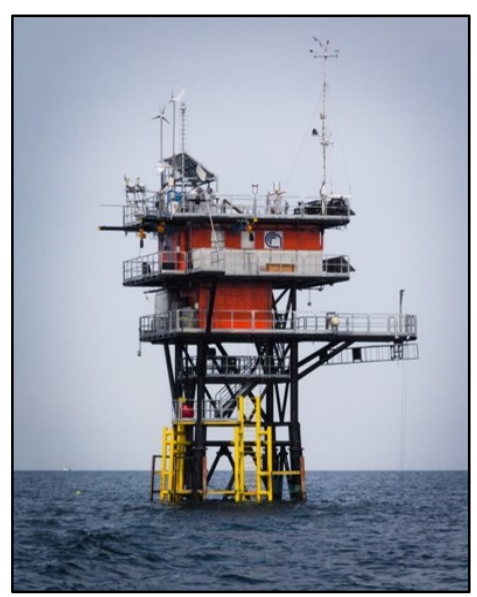

(b)

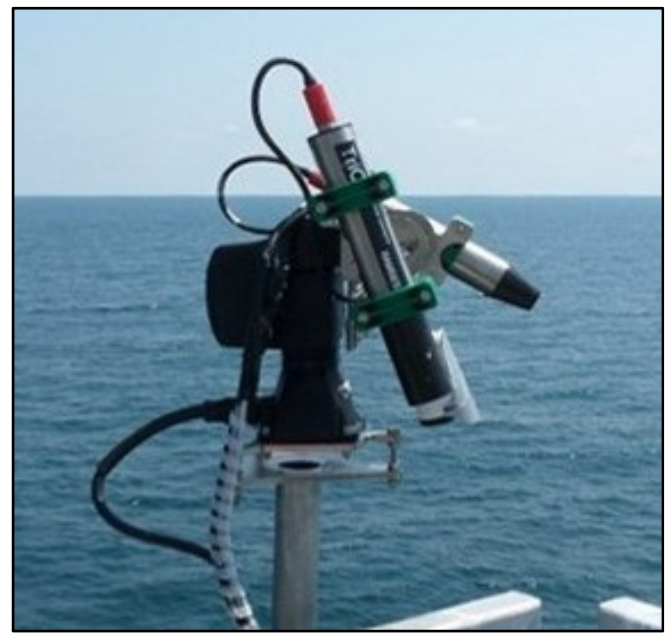

(d)

Figure 4. Photographs of PANTHYR prototype deployment at Acqua Alta Oceanographic Tower (AAOT). (a) Irradiance and sky radiance measurement position; (b) southwest face of the Acqua Alta Oceanographic Tower; (c) upwelling (water + skyglint) radiance measurement position; (d) park position. 


\subsection{Manually Supervised M3TRIOS System Used for Data Comparison}

The M3TRIOS system deployed during the FRM4SOC/FICE consists of three TRIOS RAMSES radiometers, one measuring downwelling irradiance, $E_{d}$, and two measuring radiance with fixed skyand water-viewing zenith angles of $140^{\circ}$ and $40^{\circ}$ for $L_{d}$ and $L_{u}$, respectively. The system was deployed since 2001 at many locations from both large and small ships, and platforms including AAOT [31]. Details of the instrumentation and standard measurement protocol, based on Reference [25], and data processing and quality control can be found in Reference [26]. This is quite similar to the PANTHYR processing described in Section 2.5, except for the following elements:

- The conversion from DCs to calibrated (ir)radiance is performed by the TriOS MSDA_XE software rather than the equivalent Python routines written for PANTHYR.

- Measurements are made simultaneously for $E_{d}, L_{d}$, and $L_{u}$ with a much larger number of replicate scans, at least 30, with a scan every $10 \mathrm{~s}$ for $10 \mathrm{~min}$. The first five scans passing the quality control tests described in Web Appendix 1 of Reference [26] are retained for $E_{d}, L_{d}$, and $L_{u}$

- The skyglint correction given as a quadratic function of wind speed by Reference [26] is used as an approximation of the more accurate LUT of Reference [25] described in Section 2.5 for PANTHYR.

- The skyglint correction, Equation (1), and conversion to $\rho_{w u}$, Equation (2), and subsequent NIR correction, Equations (3) and (4), are applied to each $E_{d}, L_{d}$, and $L_{u}$ triplet scan individually to give five scans for $\rho_{w}$ before mean-averaging to yield $\overline{\rho_{w}}$.

Although the instrument has wider spectral range, data are here limited to the range 400-900 nm where quality was checked by previous inter-comparison exercises. For this specific implementation, the downwelling irradiance sensor was mounted on the top deck of AAOT on a telescopic mast at height $5 \mathrm{~m}$ above deck. The sky- and water-viewing radiance sensors were mounted at the southwest face of the platform on a frame tailor-made by Tartu Observatory and Plymouth Marine Laboratory to accommodate many radiometers and to ensure $40^{\circ}$ and $140^{\circ}$ zenith-angle viewing. On the other hand, $90^{\circ}, 135^{\circ}$, or $270^{\circ}$ relative azimuth angle to sun was achieved by manual rotation of the structure before each measurement. Measurements were made typically every $30 \mathrm{~min}$ during daytime between 8:00 a.m. and 1:00 p.m. UTC.

\subsection{AERONET-OC Data Used for Comparison}

The CIMEL/Seaprism system providing data for AAOT through the AERONET-OC network was installed on the west corner of AAOT on a purpose-built jetty to minimize any shading effects of the platform on the water being measured. Full details on the instrumentation and measurement protocol and data processing and quality control can be found in Reference [6].

Level 1.5 normalized water-leaving radiance data, $L_{w n}$, were used without $f / Q$ correction [32] as it is not applied to the PANTHYR and M3TRIOS data. This data were downloaded from the AERONET-OC (AOC) website for the "Venise" site located on AAOT [6]. Level 1.5 data are automatically cloud cleared but do not have final post-deployment calibration applied and are, hence, not fully quality assured. The matchup data used in this paper do, however, pass quality control and are likely candidates for level 2.0 data if post-deployment calibration is acceptable [Zibordi, pers. comm. 18 March 2019]. Lwn values were converted to water reflectance, $\rho_{w}$, by

$$
\rho_{w}=\pi \frac{L_{w n}}{F_{0}},
$$

where $F_{0}$ is the extraterrestrial irradiance [33] resampled to gaussian band-passes with 10-nm full-width half-maximum centered on the reported exact center wavelengths of the CIMEL instrument. Each of the level 1.5 AOC measurements was matched to the closest PANTHYR measurement within 20 min, and the PANTHYR data were resampled to the same band. 


\section{Results}

\subsection{System Performance}

The system tested at AAOT performed very well and demonstrated its capability of making automated measurements following a predefined protocol and interval. After installation and configuration, there were no blocking issues while the system worked autonomously, and no intervention was required during the six days of operation while the system performed as expected.

Since the system was still in a prototype phase when tested on the AAOT, some functionality is yet to be tested or demonstrated in a real-world scenario. The system was powered from the mains grid and, as the platform was visited daily, there was no need for 3G/4G data transmission. Most measurement sites will require one or both of these functions as mains power or cabled internet may not be available.

With the defined measurement protocol and a measurement interval of $20 \mathrm{~min}$, it became obvious that the system speed needed improvements. The measurement protocol described in Section 2.4 took about 12 min to finish, depending on sun azimuth (since this affects required movements and how many measurements are skipped because they fall within the "no-go" zone). Post-FICE optimization of the control libraries for the pan/tilt head since resulted in faster movements. Additional speed can be gained in the control of the instruments, as well as by implementing multi-threading. Before each measurement cycle, some of the devices need time to start up and/or calibrate. The pan/tilt head, for example, needs to run a calibration routine to find the center point of both axes, and the IP camera needs time to boot and warm up. Implementation of multithreading would allow us to do both at the same time. Shorter measurement cycles result in more low-power standby time in between measurement cycles and lower power consumption, thus achieving better overall efficiency.

Some existing systems such as the CIMEL SeaPRISM hardware rely on the sun to calibrate the system azimuth during installation. While a high accuracy can be achieved with this method, it is only usable on sunny days. An electronic compass that achieves the required accuracy on metal structures, and that has a low power consumption, a marine-grade enclosure, and low cost is yet to be found. The current mode of operation for aligning the system zenith is achieved by placing a spirit level on the top bracket. This allows alignment as accurate as the used spirit level. After this is done, a digital inclinometer is placed on top of the instruments to verify their absolute inclination for different angles. Azimuth calibration is similar to the CIMEL system; the user can order the head to rotate toward the sun, after which the shadows cast by the top bracket serve as a reference while manually performing the alignment.

The images taken as part of each measurement cycle showed an unexpected interest from the local wildlife. Even though the system was (almost) continuously moving, birds seemed not to be deterred but showed a rather close interest in the new technology, both contaminating data by blocking the instrument field of view completely or subtly (Figure 5) and by potentially contaminating the instrument itself by fecal deposits. Major blocking of a radiometer field of view will be easily identified as bad data because the spectral signature will be different from the expected water, sky, and full sky irradiance targets. However, partial blocking of the instrument field of view (Figure 5b) may contaminate data in a way that cannot be automatically detected and rejected. Discussion with experts on bird life suggests that typical visible or audible "scarecrow" devices are not effective deterrents and the best approach is the use of spikes to prevent comfortable resting spots. We, therefore, strapped cable ties around the moving parts (Figure 4c), leaving the ends uncut as deterrent spikes, being careful to avoid the irradiance sensor field of view.

The integration of an IP camera alongside the radiometers proved particularly useful in identifying these unforeseen problems and may be useful for other causes of data contamination such as boats, floating debris/vegetation, or other unusual conditions in the radiometer field of view (Figure 5) since the radiometer gives only spatially integrated information. However, image analysis is presently subjective and is not easy to automate. 


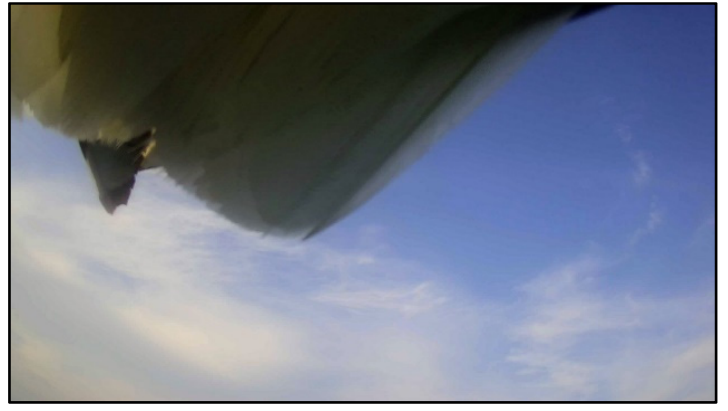

(a)

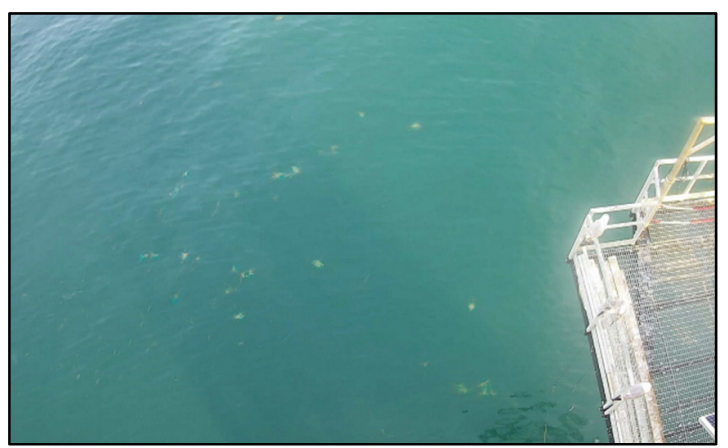

(c)

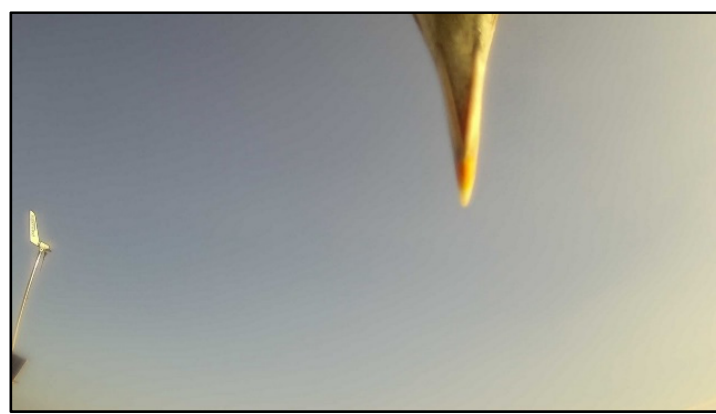

(b)

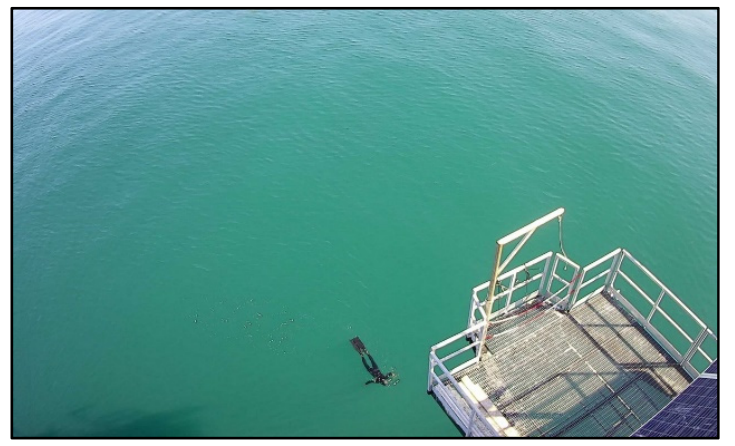

(d)

Figure 5. Sample photos taken from the camera aligned with the radiance sensor (for azimuth angles not used for measurements in this paper). (a) Bird blocking field of view; (b) more subtle measurement contamination; (c) floating vegetation and platform shadow on water; (d) unexpected water target contamination.

\subsection{Water Reflectance Spectra-Mean over Time}

For the three clear sky days analyzed here, 30 matchups were recorded between the PANTHYR and M3TRIOS systems and 10 between the PANTHYR and AERONET-OC systems. All 30 water reflectance spectra for PANTHYR and M3TRIOS systems are plotted in Figure 6, with the mean average of the 10 matchup AERONET-OC spectra. Differences between spectra observed on different days/times by a single system in this figure combine both possible temporal variability of the target itself (including possible BRDF effects, which are uncorrected for the PANTHYR and M3TRIOS systems), the random uncertainties associated with the measurement protocol and associated data processing, and certain instrument artefacts (e.g., imperfect irradiance sensor angular response). Since the intra-spectra differences are rather small, we can conclude that the water target was itself rather stable during the three days of analyzed data (spread over a six-day period). One outlier is clearly visible for the M3TRIOS system; detailed analysis of data for that measurement, including the manual log-book and various photos, suggests that this may result from an oily film visible at the water surface within the M3TRIOS system field of view. After exclusion of this outlier, the mean average water reflectance spectrum over the 29 matchups is very similar for PANTHYR and M3TRIOS systems. The mean average water reflectance spectrum over the 10 matchups is also very similar for the PANTHYR and the AERONET-OC systems. For a more detailed understanding of system data quality, including the impact of random uncertainties and removing the differences associated with water target temporal variability, the individual matchups were analyzed as described in the sections below.

\subsection{Data Comparison with M3TRIOS System-Matchup Analysis}

The 29 matchups between the PANTHYR and M3TRIOS systems, interpolated to typical multispectral wavelengths, are shown via scatterplots of $E_{d}(\lambda), L_{d}(\lambda), L_{u}(\lambda)$, and $\rho_{w}(\lambda)$, and associated 
linear regression statistics in Figure 7, including root-mean-square difference (RMSD) and mean average relative difference (MARD).
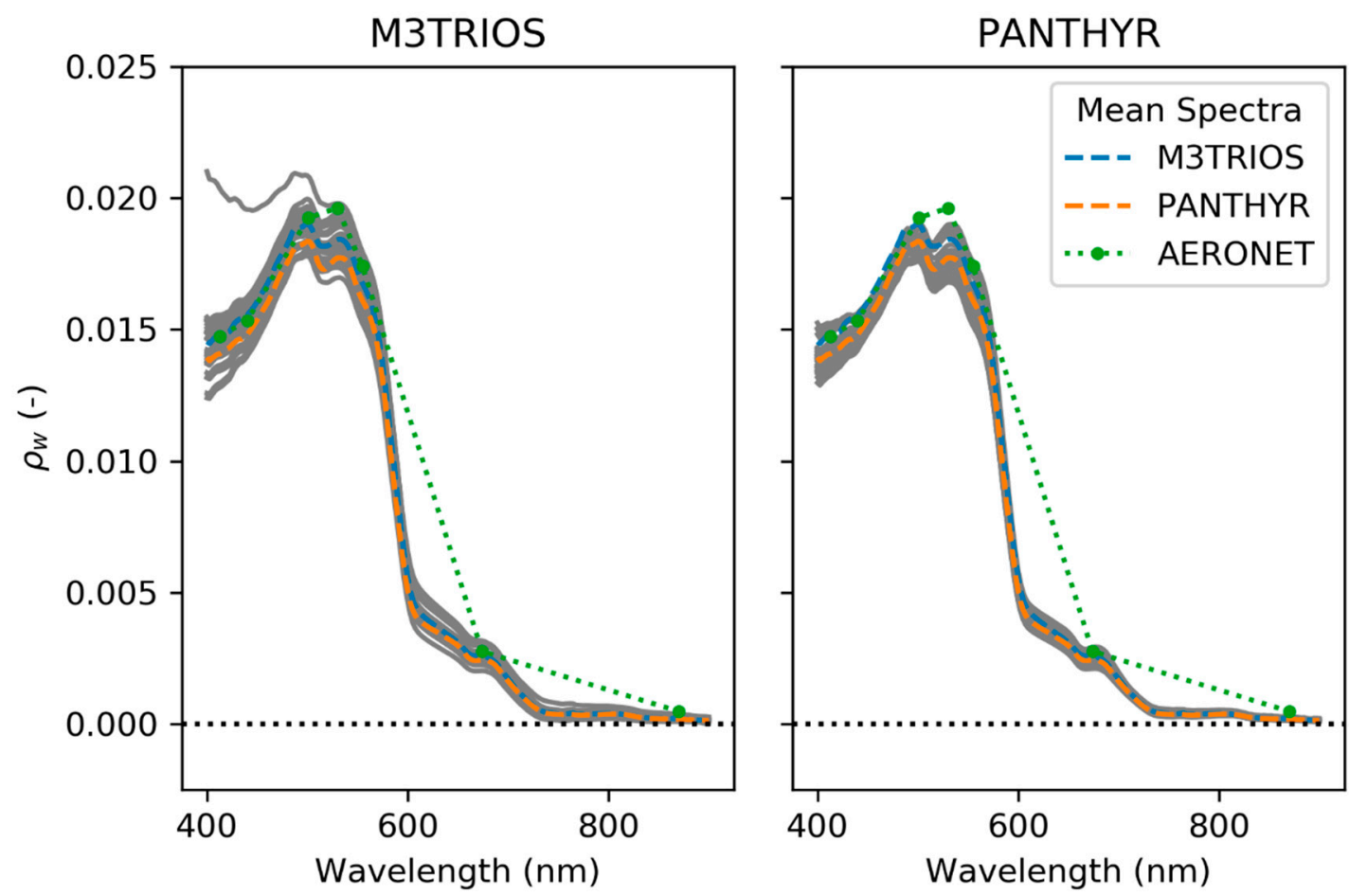

Figure 6. Water reflectance spectra for each of the 30 stations (gray lines) from (left) the three-radiometer TRIOS/RAMSES system (M3TRIOS) system and (right) the PANTHYR system. Mean spectra over all 30 stations are superimposed for the PANTHYR (orange dashed line) and the M3TRIOS system (blue dashed lines) and for all 10 measurements from the AERONET-OC system (green dotted line joining dots where the multispectral data exist).

The MARD of $E_{d}(\lambda)$ calculated over all wavelengths was 3.1\% and the scatterplot (Figure 7a) suggests some spectral variability of differences with lower PANTHYR $E_{d}(\lambda)$ for $410 \mathrm{~nm}$ and $440 \mathrm{~nm}$ and higher PANTHYR $E_{d}(\lambda)$ at $620 \mathrm{~nm}$ and $675 \mathrm{~nm}$. A full uncertainty analysis is not yet available for these instruments/systems as deployed at AAOT, and no corrections were applied for instrument artefacts such as imperfect cosine response, straylight, thermal sensitivity, non-linearity or polarization effects, or deployment imperfections, e.g., for optical perturbations from higher mast structures. Operational deployment of the PANTHYR system for fiducial reference measurements will require such an uncertainty analysis but is beyond the scope of this technology-proving paper. However, the observed differences for $E_{d}(\lambda)$ are not a cause for concern at this stage of testing. The reader is referred also to Reference [34] for a more detailed discussion of differences between measurements of $E_{d}(\lambda)$ from different instruments in typical field conditions.

The scatterplot (Figure $7 \mathrm{~b}$ ) of $L_{d}(\lambda)$ sky radiance measurements shows strong correlation but for two distinct groups of data. Analysis of the metadata recorded for both systems revealed that these distinct groups of data correspond to different azimuth angles of the PANTHYR and M3TRIOS systems; they are pointing at very different portions of the sky and, in some cases, the PANTHYR system is pointing at a much brighter sky with cirrus clouds. Since both of the obvious two groups of data corresponded to cases where PANTHYR and M3TRIOS were measuring at different azimuth angles because the systems were deployed on different sides of the AAOT platform and, hence, pointed at the water in different azimuth angles, there is no cause for concern that neither group of points has a slope of one. There were only two matchups where PANTHYR and M3TRIOS were measuring at the same azimuth angle, but this was considered insufficient for detailed analysis. 


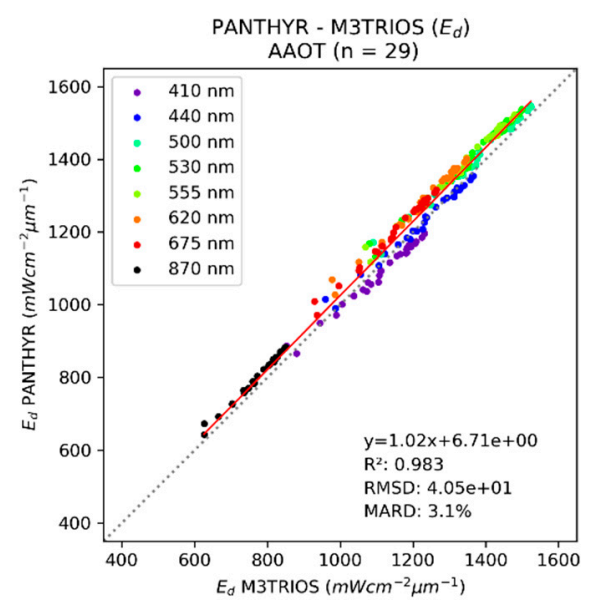

(a)

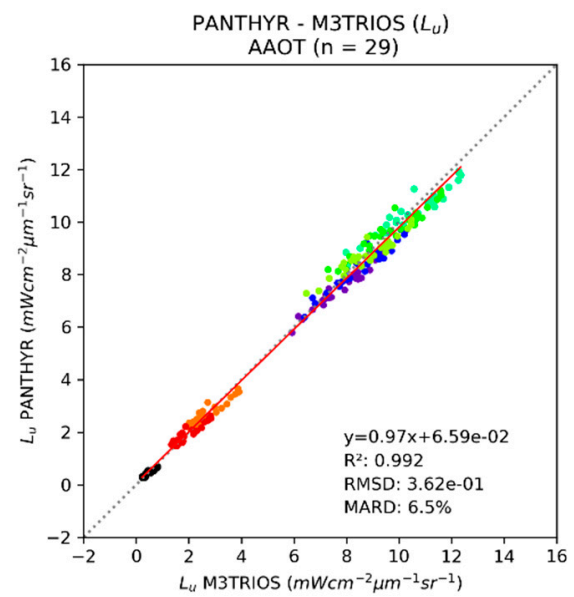

(c)

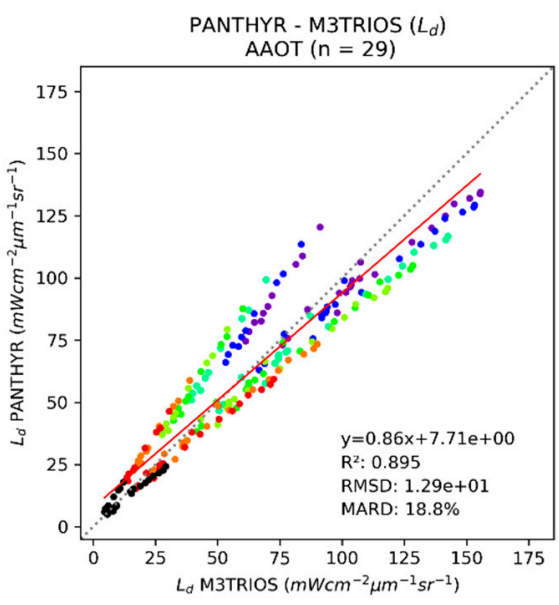

(b)

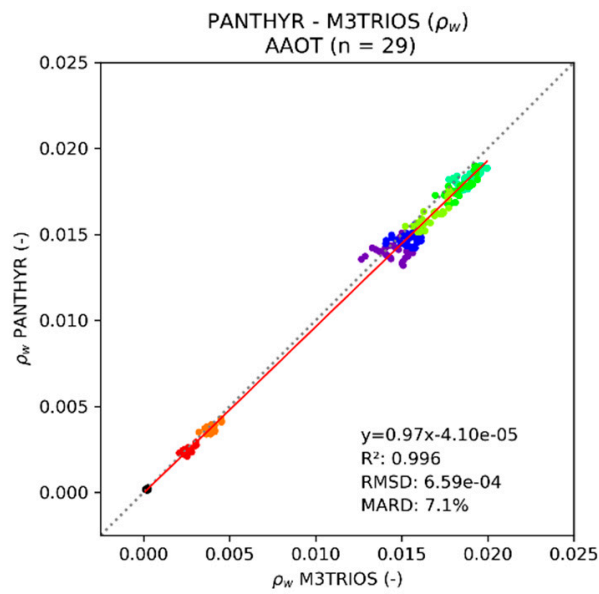

(d)

Figure 7. Scatterplot for selected wavelengths comparing PANTHYR data with M3TRIOS system data for (a) $\overline{E_{d}}$, (b) $\overline{L_{d}}$, (c) $\overline{L_{u}}$, and (d) $\overline{\rho_{w}}$ for 29 matchup stations. Linear regression statistics are given, including the root-mean-square difference (RMSD) and the mean average relative difference (MARD).

The scatterplot (Figure 7c) of $L_{u}(\lambda)$ water (plus reflected skyglint) radiance measurements shows strong correlation $\left(r^{2}=0.992\right)$ with low systematic differences (slope $\left.=0.97\right)$. The MARD of $6.5 \%$ was affected by the different azimuth angles of the two systems since this parameter includes reflected skyglint, typically $2.5-3.0 \%$ of $L_{d}(\lambda)$.

The scatterplot (Figure 7d) of $\rho_{w}(\lambda)$ water reflectance (after skyglint correction) shows high correlation between the PANTHYR and M3TRIOS systems $\left(r^{2}=0.996\right)$ with low systematic differences (slope $=0.97$ ). The MARD of $7.1 \%$ was dominated by the contribution for data at $870 \mathrm{~nm}$, where the water reflectance was very low and absolute differences, e.g., arising from skyglint correction, translated into very large relative differences.

The spectral variations of RMSD and MARD between PANTHYR and M3TRIOS for $E_{d}(\lambda)$ and $\rho_{w}(\lambda)$ over these 29 matchups are shown in Figure 8. The short wavelength scale variability for the RMSD of $E_{d}(\lambda)$ in Figure 8a was related to the similar spectral variability of $E_{d}(\lambda)$ itself, e.g., the atmospheric oxygen absorption feature at $762 \mathrm{~nm}$ or the Frauenhofer lines related to solar photosphere absorption at $516-518 \mathrm{~nm}$, and the way the slightly different central wavelengths of each $E_{d}$ instrument 
under-resolved these features. The MARD of $E_{d}(\lambda)$ between PANTHYR and M3TRIOS (Figure 8b) was $<5 \%$ for the full spectral range $400-900 \mathrm{~nm}$. The common radiometric calibration of the two $E_{d}$ instruments just prior to this experiment helped limit MARD; however, a more detailed characterization of these two instruments, particularly including an analysis of their "cosine" angular response and perhaps straylight and non-linear responses, may improve results in the future.

The spectral variation of RMSD between PANTHYR and M3TRIOS for $\rho_{w}(\lambda)$ shown in Figure 8c suggests an overall spectral shape typical of $L_{u}(\lambda)$ spectra, i.e., upwelling radiance from green water and reflected "blue" skyglint. Possible causes of this difference are multiple and certainly include imperfect correction of skyglint, but may also include different BRDF at the different azimuth angles, propagation of $E_{d}(\lambda)$ differences, $L$ radiometer calibration and characterization (straylight, non-linearity, polarization, thermal sensitivity, etc.), etc. A detailed analysis is beyond the scope of this technology-proving paper, which concludes merely that there is no major cause for concern at present.

The MARD between PANTHYR and M3TRIOS shown in Figure $8 \mathrm{~d}$ for $\rho_{w}(\lambda)$ was $<5 \%$ for the spectral range 410-580 nm, with higher MARD around $600 \mathrm{~nm}$. The much higher MARD for wavelengths higher than $700 \mathrm{~nm}$ are clearly related to the very low $\rho_{w}(\lambda)$ for these waters, showing some similarity with the pure water absorption coefficient spectrum for 700-900 nm [26] and with the phytoplankton absorption coefficient at 660-680 nm. In such conditions, the MARD has no practical relevance because a satellite validation analysis would generally consider absolute differences between PANTHYR and satellite or simply not use such data.

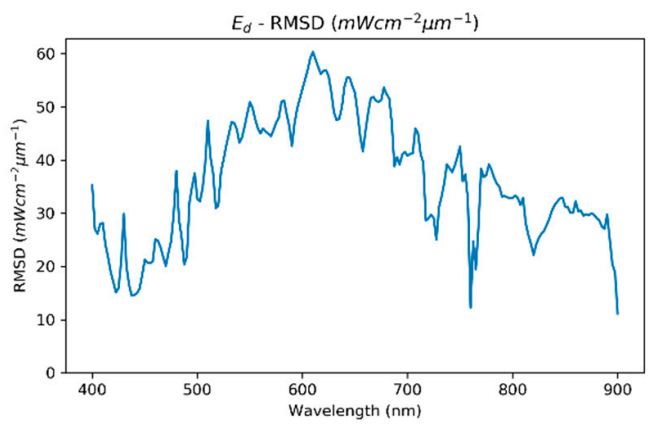

(a)

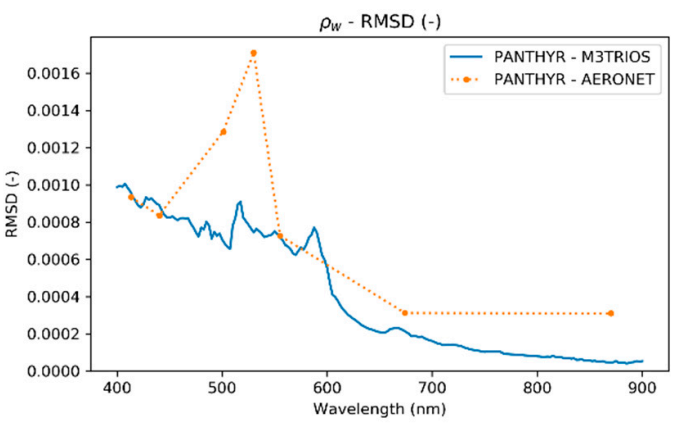

(c)

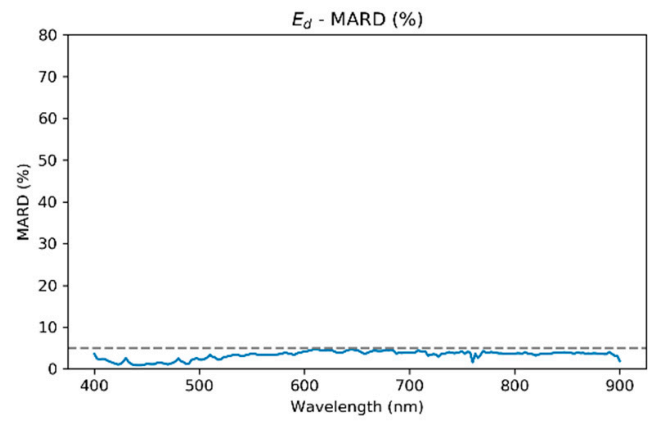

(b)

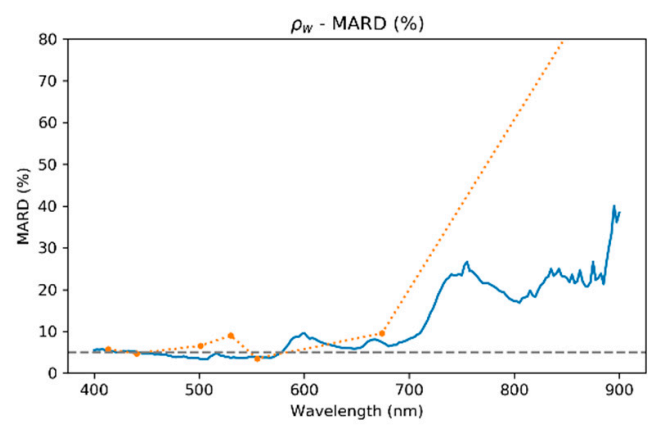

(d)

Figure 8. Root-mean-square difference (RMSD) and the mean average relative difference (MARD) between PANTHYR and M3TRIOS systems over 29 stations for $(\mathbf{a}, \mathbf{b}) \overline{E_{d}}$, and $(\mathbf{c}, \mathbf{d}) \overline{\rho_{w}}$. The 5\% MARD is shown as a horizontal dashed line.

\subsection{Data Comparison with AERONET-OC System-Matchup Analysis}

The 10 matchups between the PANTHYR and AERONET-OC systems, with resampling of PANTHYR data to gaussian spectral response functions on the AERONET-OC central wavelengths, are shown via scatterplots of $\rho_{w}(\lambda)$, and associated linear regression statistics in Figure 9. There is a strong correlation between these datasets $\left(r^{2}=0.996\right)$ with low systematic differences (slope $=0.95$ ). 
The MARD over all wavelengths appeared high (18.3\%); however, as seen in the spectral variation of MARD shown in Figure 8d, it was dominated by the data at $870 \mathrm{~nm}$, where RMSD was low but $\rho_{w}(\lambda)$ itself was very low. For the wavelengths from $413 \mathrm{~nm}$ to $555 \mathrm{~nm}$, MARD between PANTHYR and AERONET-OC was between 3.5\% and 6.5\%, except at $530 \mathrm{~nm}$, where it reached 9.0\%. Possible causes of the differences are multiple and may include imperfect correction of skyglint, propagation of $E_{d}(\lambda)$ differences (not available online for AERONET-OC), radiometer calibration and characterization (straylight and/or spectral response functions, non-linearity, polarization, thermal sensitivity, etc.), etc. A detailed analysis is beyond the scope of this technology-proving paper, which concludes merely that there is no major cause for concern at present.

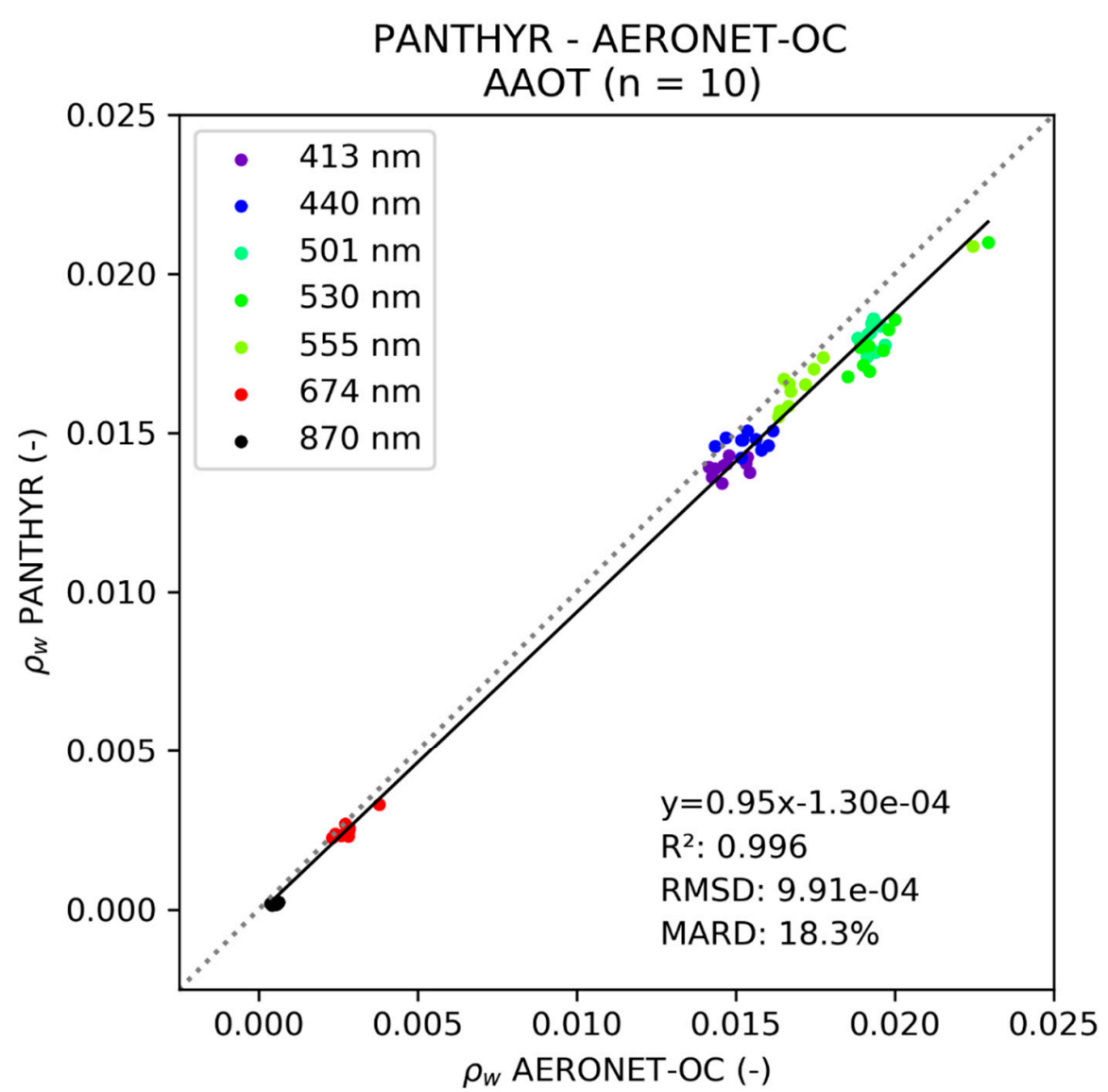

Figure 9. Scatterplot for selected wavelengths comparing PANTHYR data with AERONET-OC Venise Level 1.5 data for $\overline{\rho_{w}}$ for 10 matchups. Linear regression statistics are given, including the root-mean-square difference (RMSD) and the mean average relative difference (MARD). All PANTHYR data presented in this figure were measured at the $270^{\circ}$ relative azimuth. The black line is the reduced major axis regression. Statistics are plotted here for all wavelengths together. For per-band RMSD and MARD, see Figure 8c,d.

\section{Conclusions and Future Perspectives}

To gather enough high-quality ground-truth data for validation of water reflectance derived from satellite missions, an automated system is necessary. The AERONET-OC federated network of autonomous instrument systems [6] is now the main source of such validation data for all satellite missions measuring water reflectance but provides only multispectral data. The PANTHYR system described here aims to provide hyperspectral water reflectance data for satellite validation.

The design of the PANTHYR system was described in detail here. Two COTS hyperspectral radiometers (one radiance, one irradiance) and an IP camera are mounted on a COTS PT pointing system with original development of control electronics and software providing a low-cost, low-power, 
but robust, modular, and extendable design. Date/time/location information is received from a GNSS receiver and data are transmitted by a $4 \mathrm{G}$ gateway. A flexible mounting bracket was designed to easily fit the system to any suitable platform structure. The prototype was tested during an inter-comparison exercise organized at AAOT, an offshore platform in the Adriatic Sea. The system functioned autonomously over the six-day period without intervention. Data acquired from the system was compared with an established manually deployed hyperspectral system and with the automated AERONET-OC system data deployed simultaneously at AAOT. Data quality from the PANTHYR system was good; water reflectances compared to the two other systems had per-band MARDs within $5.5 \%$ for the spectral range $410-550 \mathrm{~nm}$ in these good measurement conditions.

Imagery from the IP camera was surprisingly useful and revealed unexpected conditions including birds within the camera field of view, which could contaminate radiometer data in significant (easily detected) or subtle (potentially undetected) ways.

The prototype tests described here were made with mains power supply and without autonomous data transmission over the internet. Use of an autonomous power supply (typically solar panels) with power supply monitoring and automated data transmission over $4 \mathrm{G}$ need to be tested in future work.

An upgrade of the PT unit to a version with slip rings is also planned to avoid the risk of cable snags possible with this first prototype.

Future mounting hardware will be machined from 5083 aluminum alloy instead of stainless steel to save weight. Lower overall weight results in lower shipping cost, and limiting the weight on top of the PT head reduces power consumption.

The system can also be extended to function on moving platforms, such as ships or buoys, via the addition of heading and inclination sensors and/or movement compensation mechanisms.

The data acquisition and processing described here are based strongly on precursor work and provide a robust starting point. However, future research may take advantage of the flexible pointing capability to investigate improved data acquisition protocols, e.g., with multiple zenith angle radiance measurements. The skyglint correction for above-water reflectance measurements is also considered to be a major source of measurement uncertainty, and potential improvements were investigated in many recent studies. When considered mature, such improvements can easily be incorporated in the processing software described here.

In conclusion, the PANTHYR system prototype was successfully tested in a basic configuration (without autonomous power supply and without data transmission over the internet) giving robust performance and high-quality hyperspectral data. The system prototype meets the requirements for future worldwide deployment in a network for hyperspectral validation of water reflectance data from satellites.

Author Contributions: Conceptualization, D.V., K.R., and A.C.; methodology, D.V., K.R., and A.C.; software, D.V. and Q.V.; validation, Q.V. and M.B.; formal analysis, K.R., Q.V., and M.B.; writing-original draft preparation, K.R. and D.V.; writing-review and editing, A.C., Q.V., M.B., and D.V.

Funding: This research was funded by BELSPO (Belgian Science Policy Office) in the framework of the STEREO III program project HYPERMAQ (SR/00/335), and by the European Space Agency PRODEX/HYPERNET-OC project. The AAOT fieldwork was partially funded by the European Space Agency FRM4SOC project.

Acknowledgments: The authors would like to thank the Plymouth Marine Laboratory (PML) and Consiglio Nazionale delle Ricerche (CNR), Dr. Gavin Tilstone, Tartu Observatory, and the FRM4SOC project for organizing the FICE at AAOT and for providing mounting frames for the M3TRIOS system radiometers. Tartu Observatory provided the radiometric calibration of all five RAMSES instruments used here. Dr. Giuseppe Zibordi is acknowledged for providing the AERONET-OC data and for kind advice. TRIOS Gmbh is acknowledged for providing details of the serial protocol communication for the RAMSES instruments, allowing us to write the necessary data acquisition software. For the latter, the RFLEX code provided by Stefan Simis provided some useful ideas on how to proceed. Michiel T'Jampens provided appreciated technical feedback. We thank the three anonymous reviewers for many constructive comments on the first version of this paper.

Conflicts of Interest: The authors declare no conflicts of interest. The funders had no role in the design of the study; in the collection, analyses, or interpretation of data; in the writing of the manuscript, or in the decision to publish the results. 


\section{References}

1. Gohin, F.; Saulquin, B.; Oger-Jeanneret, H.; Lozac'h, L.; Lampert, L.; Lefebvre, A.; Riou, P.; Bruchon, F. Towards a better assessment of the ecological status of coastal waters using satellite-derived chlorophyll-a concentrations. Remote Sens. Environ. 2008, 112, 3329-3340. [CrossRef]

2. Bresciani, M.; Stroppiana, D.; Odermatt, D.; Morabito, G.; Giardino, C. Assessing remotely sensed chlorophyll-a for the implementation of the Water Framework Directive in European perialpine lakes. Sci. Total Environ. 2011, 409, 3083-3091. [CrossRef] [PubMed]

3. Vanhellemont, Q.; Ruddick, K.G. Turbid wakes associated with offshore wind turbines observed with Landsat 8. Remote Sens. Environ. 2014, 145, 105-115. [CrossRef]

4. Giardino, C.; Brando, V.E.; Gege, P.; Pinnel, N.; Hochberg, E.; Knaeps, E.; Reusen, I.; Doerffer, R.; Bresciani, M.; Braga, F.; et al. Imaging Spectrometry of Inland and Coastal Waters: State of the Art, Achievements and Perspectives. Surv. Geophys. 2018, 40, 401-429. [CrossRef]

5. Bailey, S.W.; Werdell, P.J. A multi-sensor approach for the on-orbit validation of ocean color satellite data products. Remote Sens. Environ. 2006, 102, 12-23. [CrossRef]

6. Zibordi, G.; Holben, B.; Slutsker, I.; Giles, D.; D’Alimonte, D.; Mélin, F.; Berthon, J.-F.; Vandemark, D.; Feng, H.; Schuster, G.; et al. AERONET-OC: A network for the validation of ocean color primary product. J. Atmos. Ocean. Technol. 2009, 26, 1634-1651. [CrossRef]

7. Hlaing, S.; Harmel, T.; Gilerson, A.; Foster, R.; Weidemann, A.; Arnone, R.; Wang, M.; Ahmed, S. Evaluation of the VIIRS ocean color monitoring performance in coastal regions. Remote Sens. Environ. 2013, 139, 398-414. [CrossRef]

8. Mélin, F.; Sclep, G. Band shifting for ocean color multi-spectral reflectance data. Opt. Express 2015, 23, 2262-2279. [CrossRef]

9. Holben, B. AERONET-A federated instrument network and data archive for aerosol characterization. Remote Sens. Environ. 1998, 66, 1-16. [CrossRef]

10. Zibordi, G.; Mélin, F.; Hooker, S.B.; D'Alimonte, D.; Holben, B. An autonomous above-water system for the validation of ocean colour radiance data. IEEE TGARS 2004, 42, 401-415.

11. Barreto, Á.; Cuevas, E.; Granados-Muñoz, M.-J.; Alados-Arboledas, L.; Romero, P.M.; Gröbner, J.; Kouremeti, N.; Almansa, A.F.; Stone, T.; Toledano, C.; et al. The new sun-sky-lunar Cimel CE318-T multiband photometer \&ndash; a comprehensive performance evaluation. Atmos. Meas. Tech. 2016, 9, 631-654. [CrossRef]

12. Hooker, S.B.; Bernhard, G.; Morrow, J.H.; Booth, C.R.; Comer, T.; Lind, R.N.; Quang, V. Optical Sensors for Planetary Radiant Energy (Osprey): Calibration and Validation of Current and Next-Generation Nasa Missions; NASA Tech. Memo. 2011-215872; NASA Goddard Space Flight Center: Greenbelt, MD, USA, 2012.

13. Simis, S.G.H.; Olsson, J. Unattended processing of shipborne hyperspectral reflectance measurements. Remote Sens. Environ. 2013, 135, 202-212. [CrossRef]

14. Slivkoff, M.M. Ocean Colour Remote Sensing of the Great Barrier Reef Water. Ph.D. Thesis, Department of Imaging \& Applied Physics, School of Science, Curtin University, Curtin, Australia, 2014.

15. Brando, V.; Lovell, J.; King, E.; Boadle, D.; Scott, R.; Schroeder, T. The Potential of Autonomous Ship-Borne Hyperspectral Radiometers for the Validation of Ocean Color Radiometry Data. Remote Sens. 2016, 8, 150. [CrossRef]

16. Carswell, T.; Costa, M.; Young, E.; Komick, N.; Gower, J.; Sweeting, R. Evaluation of MODIS-Aqua Atmospheric Correction and Chlorophyll Products of Western North American Coastal Waters Based on 13 Years of Data. Remote Sens. 2017, 9, 1063. [CrossRef]

17. Hommersom, A.; Kratzer, S.; Laanen, M.; Ansko, I.; Ligi, M.; Bresciani, M.; Giardino, C.; Betlrán-Abaunza, J.M.; Moore, G.; Wernand, M.R.; et al. Intercomparison in the field between the new WISP-3 and other radiometers (TriOS Ramses, ASD FieldSpec, and TACCS). J. Appl. Remote Sens. 2012, 6, 063615. [CrossRef]

18. Ruddick, K.; De Cauwer, V.; Park, Y.; Becu, G.; De Blauwe, J.-P.; Vreker, E.D.; Deschamps, P.-Y.; Knockaert, M.; Nechad, B.; Pollentier, A.; et al. Preliminary Validation of MERIS Water Products for Belgian Coastal Waters; European Space Agency: Paris, France, 2003; Volume SP-531.

19. Talone, M.; Zibordi, G.; Ansko, I.; Banks, A.C.; Kuusk, J. Stray light effects in above-water remote-sensing reflectance from hyperspectral radiometers. Appl. Opt. 2016, 55, 3966. [CrossRef] [PubMed]

20. Zibordi, G.; Talone, M.; Jankowski, L. Response to Temperature of a Class of In Situ Hyperspectral Radiometers. J. Atmos. Ocean. Technol. 2017, 34, 1795-1805. [CrossRef] 
21. Talone, M.; Zibordi, G. Polarimetric characteristics of a class of hyperspectral radiometers. Appl. Opt. 2016, 55, 10092. [CrossRef] [PubMed]

22. Mekaoui, S.; Zibordi, G. Cosine error for a class of hyperspectral irradiance sensors. Metrologia 2013, 50, 187-199. [CrossRef]

23. Olesen, D.; Jakobsen, J.; Knudsen, P. Knudsen Low-cost GNSS sampler based on the beaglebone black SBC. In Proceedings of the 2016 8th ESA Workshop on Satellite Navigation Technologies and European Workshop on GNSS Signals and Signal Processing (NAVITEC), Noordwijk, The Netherlands, 14-16 December 2016; pp. 1-7.

24. Mollon, M.; Kaneko, E.H.; Niro, L.; Montezuma, M. Remote Laboratory for a Servomotor Control System with Embedded Architecture; IJAREEIE: Chennai, Tamilnadu, India, 2017; Volume 6.

25. Mobley, C.D. Estimation of the remote-sensing reflectance from above-surface measurements. Appl. Opt. 1999, 38, 7442-7455. [CrossRef] [PubMed]

26. Ruddick, K.; De Cauwer, V.; Park, Y.; Moore, G. Seaborne measurements of near infrared water-leaving reflectance: The similarity spectrum for turbid waters. Limnol. Oceanogr. 2006, 51, 1167-1179. [CrossRef]

27. Doyle, J.P.; Zibordi, G. Optical propagation within a three-dimensional shadowed atmosphere-ocean field: Application to large deployment structures. Appl. Opt. 2002, 41, 4283-4306. [CrossRef] [PubMed]

28. Ruddick, K.; Cauwer, V.D.; Van Mol, B. Use of the Near Infrared Similarity Spectrum for the Quality Control of Remote Sensing Data; Frouin, R.J., Babin, M., Sathyendranath, S., Eds.; SPIE: Bellingham, WA, USA, 2005; Volume 5885.

29. Zibordi, G.; Berthon, J.F.; Doyle, J.P.; Grossi, S.; van der linde, D.; Targa, C.; Alberotanza, L. Coastal Atmosphere and Sea Time Series (CoASTS), Part 1: A Long-Term Measurement Program; Tech Memo TM-2002-206892; NASA Goddard Space Flight Center: Greenbelt, MD, USA, 2002.

30. Hooker, S.B.; Lazin, G.; Zibordi, G.; McLean, S. An evaulation of above- and in-water methods for determining water-leaving radiances. J. Atmos. Ocean. Technol. 2002, 19, 486-515. [CrossRef]

31. Zibordi, G.; Ruddick, K.; Ansko, I.; Moore, G.; Kratzer, S.; Icely, J.; Reinart, A. In situ determination of the remote sensing reflectance. Ocean Sci. 2012, 8, 567-586. [CrossRef]

32. Morel, A.; Antoine, D.; Gentili, B. Bidirectional reflectance of oceanic waters: Accounting for Raman emission and varying particle scattering phase function. Appl. Opt. 2002, 41, 6289-6306. [CrossRef] [PubMed]

33. Thuillier, G.; Herse, M.; Labs, D.; Foujols, T.; Peetermans, W.; Gillotay, D.; Simon, P.C.; Mandel, H. The solar spectral irradiance from 200 to $2400 \mathrm{~nm}$ as measured by the SOLSPEC spectrometer from the ATLAS and EURECA missions. Sol. Phys. 2003, 214, 1-22. [CrossRef]

34. Vabson, V.; Kuusk, J.; Ansko, I.; Vendt, R.; Alikas, K.; Ruddick, K.; Ansper, A.; Bresciani, M.; Burmester, H.; Costa, M.; et al. Field intercomparison of radiometers used for satellite validation in the 400-900 nm range. Remote Sens. 2019, 11, 1129. [CrossRef]

(C) 2019 by the authors. Licensee MDPI, Basel, Switzerland. This article is an open access article distributed under the terms and conditions of the Creative Commons Attribution (CC BY) license (http://creativecommons.org/licenses/by/4.0/). 